\title{
The Explorer of Diffuse Galactic Emission (EDGE)
}

\author{
R. F. Silverberg ${ }^{1}$, E. S. Cheng ${ }^{2}$, D. A. Cottingham ${ }^{3}$, D. J. Fixsen ${ }^{4}$, \\ L. $\mathrm{Knox}^{5}$, S. S. Meyer ${ }^{6}$, P. T. Timbie ${ }^{7}$, and G. W. Wilson ${ }^{8}$ \\ ${ }^{1}$ NASA/Goddard Space Flight Center, Greenbelt, MD 20771, USA \\ ${ }^{2}$ Conceptual Analytics, Glenn Dale, MD 20769, USA \\ ${ }^{3}$ Global Science and Technology, Greenbelt, MD 20771 USA \\ ${ }^{4}$ SSAI, Lanham, MD 20706 USA \\ ${ }^{5}$ University of California at Davis, Davis, CA 95616 USA \\ ${ }^{6}$ Enrico Fermi Institute, University of Chicago, Chicago, IL 60637 USA \\ ${ }^{7}$ University of Wisconsin, Madison, WI 53706 USA \\ ${ }^{8}$ University of Massachusetts, Amherst, MA 01003 USA
}

\section{Summary}

The details of the formation of the first objects, stars and galaxies and their subsequent evolution remain a cosmological unknown. Few observational probes of these processes exist. The Cosmic Infrared Background (CIB) originates from this era and measurements of its anisotropy can provide information to test models of both galaxy evolution and the growth of primordial structure. Such measurements should provide a sensitive probe of the large-scale variation in protogalaxy density at redshifts, $z \sim 0.5-3$, while optical galaxy surveys provide complementary information at $z<0.5$ and Lyman alpha absorption forest studies and Cosmic Microwave Background measurements add information at higher redshifts.

The Explorer of Diffuse Galactic Emission (EDGE) is a balloon-borne mission designed to measure the spatial fluctuations in the CIB from $200 \mu \mathrm{m}$ to $1 \mathrm{~mm}$ on $6^{\prime}$ to $3^{\circ}$ scales with up to $2 \mu \mathrm{K}$ sensitivity/resolution element on an Antarctic Long Duration Balloon (LDB) flight. EDGE will employ an array of Frequency Selective Bolometers (Kowitt et al., 1996) to simultaneously measure spectral and spatial information with seven sky pixels each containing eight spectral bands. EDGE is designed to cover $\sim 100$ square degrees of the sky in a single $\sim 10$ day LDB flight to create a new observational constraint for large-scale structure formation theories.

\section{References}

Kowitt, M. S., Fixsen, D. J., Goldin, A., \& Meyer, S. S., 1996, Appl. Optics, 35, 5630 